\title{
Nefropatia induzida por contraste: avaliação da proteção pela n-acetilcisteína e alopurinol em ratos uninefrectomizados*
}

\author{
Contrast-induced nephropathy: evaluation of $n$-acetylcysteine and allopunirol protective \\ effect in uninephrectomized rats
}

José Carlos Carraro Eduardo ${ }^{1}$, Heloisa Werneck de Macedo², Maria Lucia Ribeiro Caldas ${ }^{3}$, Licio Esmeraldo Silva ${ }^{4}$

Resumo OBJETIVO: A nefropatia por contraste é a terceira causa de insuficiência renal aguda em pacientes hospitalizados. $O$ objetivo deste estudo foi avaliar a ação da n-acetilcisteína e do alopurinol na proteção renal em ratos de ambos os sexos que receberam diatrizoato. MATERIAIS E MÉTODOS: Ratos Wistar adultos jovens, uninefrectomizados e submetidos a restrição hídrica, receberam solução salina (grupo 1: machos; grupo 2: fêmeas), diatrizoato (grupo 3: machos; grupo 4: fêmeas), diatrizoato e n-acetilcisteína (grupo 5: machos), diatrizoato e alopurinol (grupo 6: machos) e diatrizoato e n-acetilcisteína + alopurinol (grupo 7: machos). A filtração glomerular foi avaliada pela creatinina. 0 teste $t$ de Student e o teste do sinal foram utilizados para análises estatísticas. RESULTADOS: Ratos que receberam diatrizoato apresentaram elevação estatisticamente significante da creatinina sérica, quando comparados aos controles, porém não houve diferença entre os sexos. Os animais que receberam alopurinol não mostraram aumento significante da creatinina, enquanto a administração de n-acetilcisteína não impediu a elevação da creatinina. CONCLUSÃO: 0 alopurinol mostrouse mais efetivo que a n-acetilcisteína na proteção funcional renal ao dano induzido pelo diatrizoato de sódio. Não houve diferença entre os sexos na intensidade do dano renal pelo diatrizoato de sódio.

Unitermos: Nefropatia induzida por contraste; N-acetilcisteína; Alopurinol; Diatrizoato.

Abstract OBJECTIVE: Contrast medium-induced nephropathy is the third most frequent cause of iatrogenic acute renal failure involving inpatients. The present study was aimed at evaluating the protective effect of n-acetylcysteine and allopurinol in both male and female rats receiving diatrizoate. MATERIALS AND METHODS: Thirty-five young adult Wistar rats submitted to hydric restriction were divided into groups as follows: groups 1 and 2 (respectively male and female rats) receiving saline solution; groups 3 and 4 (respectively male and female rats) receiving diatrizoate; group 5 (male rats) receiving diatrizoate and $n$-acetylcysteine; group 6 (male rats) receiving diatrizoate and allopurinol; and group 7 (male rats) receiving diatrizoate and $n$-acetylcysteine + allopurinol. The glomerular filtration was evaluated by measurement of creatinine clearance. Student's $t$-test and the test of signal were utilized for statistical analysis. RESULTS: Animal models receiving allopurinol did not present a significant increase in the creatinine levels, while n-acetylcysteine did not prevent the creatinine levels increase. CONCLUSION: Allopurinol has shown to be more effective than $n$-acetylcysteine in the renal function protection against sodium diatrizoate-induced damages. No difference has been found between male and female groups as regards the intensity of sodium diatrizoate-induced renal damages.

Keywords: Contrast-induced nephropathy; N-acetylcysteine; Allopurinol; Diatrizoate.

Carraro-Eduardo JC, Macedo HW, Caldas MLR, Silva LE. Nefropatia induzida por contraste: avaliação da proteção pela nacetilcisteína e alopurinol em ratos uninefrectomizados. Radiol Bras. 2008;41(3):177-181.

* Trabalho realizado na Faculdade de Medicina da Universidade Federal Fluminense (UFF), Niterói, RJ, Brasil.

1. Mestre, Docente da Faculdade de Medicina da Universidade Federal Fluminense (UFF), Niterói, RJ, Brasil.

2. Doutora, Subcoordenadora de Pós-Graduação em Patologia da Faculdade de Medicina da Universidade Federal Fluminense (UFF), Niterói, RJ, Brasil.

3. Doutor, Chefe do Serviço de Patologia Renal do Hospital Universitário Antônio Pedro (HUAP) da Faculdade de Medicina da Universidade Federal Fluminense (UFF), Niterói, RJ, Brasil.

4. Mestre, Docente do Instituto de Matemática da Universidade Federal Fluminense (UFF), Niterói, RJ, Brasil.

Endereço para correspondência: Dr. José Carlos Carraro Eduardo. Estrada Francisco da Cruz Nunes, 8100 , casa 503, Itaipu. Niterói, RJ, Brasil, 24350-310. E-mail: carraro@urbi.com.br

\section{INTRODUÇÃO}

Os meios de contraste iodados são imprescindíveis na prática médica atual. Estima-se em mais de 80 milhões/ano o número de intervenções diagnósticas ou terapêuticas que são realizadas com o auxílio de meios de contraste, no mundo ${ }^{(1)}$. Nos

Recebido para publicação em 10/9/2007. Aceito, após revisão, em 1/10/2007.
Estados Unidos, o número de cateterizações cardíacas e procedimentos invasivos coronarianos percutâneos no ano de 2003 foi de mais dois milhões, o que representa um aumento superior a $300 \%$ nas duas últimas décadas ${ }^{(2)}$.

A nefropatia induzida por radiocontraste (NIC) é definida por aumento absoluto igual ou maior que $0,5 \mathrm{mg} / \mathrm{dl}$ ou por aumento relativo maior que $25 \%$ no valor basal da creatinina ocorrendo nas primeiras 
48 horas após a exposição a um contraste radiológico endovenoso ${ }^{(3)}$. A NIC é a terceira causa iatrogênica de insuficiência renal aguda em pacientes hospitalizados e está associada com taxa de mortalidade elevada - acima de $34 \%$ — nesta população ${ }^{(4,5)}$

A incidência da NIC é variável e depende da presença de fatores de risco, do tipo e da quantidade do meio de contraste utilizado e da sensibilidade do método empregado para o diagnóstico do acometimento renal ${ }^{(6)}$. Em estudos prospectivos, a incidência da NIC variou entre $12 \%$ e $27 \%$, porém pode chegar a mais de $60 \%$ em pacientes portadores de nefropatia diabética com creatinina acima de $3 \mathrm{mg} / \mathrm{dl}^{(7)}$. Outros fatores, como idade avançada, insuficiência cardíaca, hipovolemia, insuficiência renal, hepatopatia crônica e doses elevadas de contraste, são freqüentemente concomitantes e obrigam a uma avaliação crítica de riscos e benefícios para a realização de exames contrastados. Quando exames utilizando meios de contraste forem imprescindíveis, medidas protetoras devem ser utilizadas ${ }^{(6,8,9)}$

Recentemente, o sexo feminino ${ }^{(\mathbf{1 0})}$ e a hiperuricemia $^{(11)}$ têm sido apontados como fatores de risco para a NIC em pacientes com doença renal crônica. A opção com exames de ressonância magnética que utilizam o gadolínio, um agente que não está relacionado ao desenvolvimento de NIC, deve ser ponderada criteriosamente, após as publicações recentes relacionando o aparecimento de dermopatia fibrosante nefrogênica com o uso do gadolínio em pacientes com insuficiência renal crônica ${ }^{(\mathbf{1 2})}$

Os agentes de contraste diferem significativamente com respeito às suas propriedades físicas e bioquímicas. Importantes propriedades que podem influenciar a relativa eficácia e efetividade destes compostos incluem a ionicidade, a osmolalidade, a estrutura química e a viscosidade. Apesar da grande expectativa com o surgimento dos contrastes de baixa osmolalidade e os não-iônicos isosmolares, menos tóxicos que os contrastes hiperosmolares, esses compostos não se mostraram capazes de prevenir a nefropatia induzida por meios de contraste $^{(\mathbf{7 , 9 )}}$. Mais recentemente, ênfase vem sendo dada à viscosidade desses compostos, o que poderia explicar por que o iodixanol, um composto isosmolar com alta viscosidade, tenha falhado na prevenção da NIC ${ }^{(2)}$.

Vacuolização das células epiteliais dos túbulos proximais renais se segue à administração do contraste intravascular e as alterações estruturais são reversíveis após alguns dias da administração do agente de contraste. Parece não haver correlação entre o grau de vacuolização nas células tubulares e a intensidade do dano funcional renal $^{(3)}$.

Diversos mecanismos têm sido propostos para a NIC, especialmente a vasoconstrição na medular renal e conseqüentes redução do fluxo sanguíneo medular e isquemia tubular renal, que se somariam à citotoxicidade direta ${ }^{(3,8)}$. Além destes potenciais mecanismos, a participação de espécies reativas do oxigênio em decorrência da produção de radicais livres tem sido apontada como de grande importância para a instalação da NIC ${ }^{(13)}$. Estudos experimentais em ratos com depleção de volume indicam que os agentes de contraste reduzem a atividade de enzimas antioxidantes na cortical renal $^{(\mathbf{1 4})}$.

Em razão da crescente necessidade do uso de agentes de contraste iodados em pacientes portadores de fatores de risco para a NIC, do reconhecimento de que os novos agentes, embora menos tóxicos, não previnem a NIC, e do melhor conhecimento da fisiopatologia do dano renal que se segue ao uso intravenoso desses compostos, diversas drogas têm sido testadas para atenuar ou impedir o dano renal induzido pelos radiocontrastes. Entre essas drogas estão a dopamina, o manitol, a endotelina, os bloqueadores dos canais de cálcio, os "varredores de radicais livres" e o fenoldo-

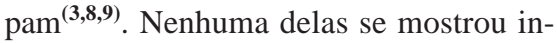
questionavelmente superior à hidratação.

A n-acetilcisteína, por suas propriedades antioxidantes, por ser livremente filtrada e com fácil acesso aos compartimentos intracelulares, e pela sua segurança no uso clínico é a droga mais estudada e a que resultados mais promissores tem mostrado ${ }^{(\mathbf{1 5 - 2 0})}$. O alopurinol, um inibidor da xantina-oxidase, tem sido amplamente utilizado na prática médica no tratamento dos estados hiperuricêmicos, nos últimos 50 anos. Mais recentemente, estudos experimentais têm demonstrado um possível papel do alopu- rinol na prevenção da nefropatia associada aos processos de isquemia/reperfusão, cuja fisiopatologia envolve a produção de

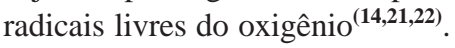

Este trabalho tem como objetivo avaliar o papel da n-acetilcisteína e do alopurinol na proteção renal em ratos de ambos os sexos submetidos à administração venosa de diatrizoato de sódio.

\section{MATERIAIS E MÉTODOS}

\section{Animais}

Ratos Wistar jovens machos e fêmeas (200-250 g) foram alojados em sala refrigerada com 12 horas de luz e temperatura $\left(22 \pm 2{ }^{\circ} \mathrm{C}\right)$ e umidade relativa constantes. Os ratos foram alojados de acordo com os grupos de tratamento, com cinco animais do mesmo sexo por gaiola de fundo de plástico com leito de maravalha esterilizada e recebendo água e ração padronizada $a d l i$ bitum. Permaneceram em período de adaptação por duas semanas. Foram então submetidos a nefrectomia esquerda sob anestesia com ketamina $50 \mathrm{mg} / \mathrm{kg}$ e xilazina 8 $\mathrm{mg} / \mathrm{kg}$ por via intramuscular.

\section{Protocolo experimental}

No $15^{\circ}$ dia após a nefrectomia os animais foram submetidos a privação de água por 24 horas e receberam as drogas conforme os seguintes grupos: G1 - machos, salina; G2 - fêmeas, salina; G3 - machos, diatrizoato; G4 - fêmeas, diatrizoato; G5 - machos, diatrizoato + n-acetilcisteína; G6 - machos, diatrizoato + alopurinol; G7 machos, diatrizoato $+n$-acetilcisteína + alopurinol. O diatrizoato de sódio foi administrado apenas uma vez, no $17^{\circ}$ dia pósnefrectomia. A n-acetilcisteína e o alopurinol foram administrados no $16^{\circ}$ e $17^{\circ}$ dias pós-nefrectomia. Amostras de sangue foram obtidas por punção cardíaca sob anestesia, imediatamente antes do início da administração das drogas e ao final do tratamento, para dosagem da creatinina.

\section{Drogas}

$\mathrm{N}$-acetilcisteína (Fluimucil ${ }^{\circledR}, 600 \mathrm{mg}$ [Zambon; São Paulo, Brasil]), 300 mg/kg, em suspensão com 60 mg/ml, preparada em solução fisiológica, intraperitoneal; alopurinol (Zyloric ${ }^{\circledR} 100$ mg/comprimido [Glaxo Welcome; Rio de Janeiro, Brasil]), 
$150 \mathrm{mg} / \mathrm{kg}$, em suspensão com $25 \mathrm{mg} / \mathrm{ml}$, preparada em solução fisiológica, intraperitoneal; diatrizoato de sódio (Hypaque ${ }^{\circledR}$ $76 \%$ [Sanofi Winthrop; Rio de Janeiro, Brasil]), dose única de $1,9 \mathrm{ml} / \mathrm{kg}$ (2,9 g iodo/ $\mathrm{kg}$ ), veia caudal.

\section{Análise estatística dos dados}

Os dados foram descritos estatisticamente em termos de média \pm desvio-padrão. O teste de Shapiro-Wilk avaliou a normalidade dos dados. Comparações de grupos pareados normais (medidas pré e pós-aplicação de um tratamento) utilizaram o teste $t$ pareado. Quando os dados não se mostraram normais, utilizou-se o teste do sinal. O nível de significância utilizado foi de 0,05 .

\section{RESULTADOS}

Na Tabela 1 estão apresentados os resultados das dosagens de creatinina (média \pm desvio-padrão) de machos e fêmeas tratados com salina ou diatrizoato. A administração de solução salina em animais machos não determinou elevação da creatinina $(0,45 \pm 0,09$ e $0,58 \pm 0,03$, pré e pós, respectivamente; $t=2,017$; valor- $p=$ $0,107)$, enquanto os animais do mesmo sexo que receberam o diatrizoato apresentaram creatinina sérica elevada $(0,56 \pm 0,03$ e $0,87 \pm 0,08 ; t=8,348$; valor- $p=0,001$ ) As fêmeas que receberam salina não apresentaram elevação da creatinina $(0,61 \pm 0,1$ e $0,63 \pm 0,09 ; t=0,844$; valor- $p=0,421$ ) Fêmeas tratadas com diatrizoato tiveram elevação significativa da creatininemia $(0,46 \pm 0,16$ e $0,78 \pm 0,13 ; t=4,867$; valor- $p=0,004)$. Não houve diferença significativa $(p>0,05)$, na comparação entre machos e fêmeas, quanto ao dano renal avaliado por meio da dosagem de creatinina.

A administração de n-acetilcisteína em ratos machos tratados com diatrizoato de sódio não evitou a elevação estatisticamente significante da creatinina $(0,50 \pm$ 0,07 antes da administração da droga e 0,72 \pm 011 após; $t=4,674$; valor- $p=0,0002$ ). Já a administração de alopurinol não foi acompanhada de acréscimo significativo da creatininemia durante o tratamento com o diatrizoato de sódio $(0,54 \pm 0,18$ antes e $0,64 \pm 0,15$ depois; teste do sinal: valor- $p$ $=0,688)$. A associação das drogas n-ace-

Tabela 1 Valores de creatinina em mg/dl (média \pm desvio-padrão) nos grupos salina e diatrizoato de sódio, pré e pós-tratamento, em machos e fêmeas.

\begin{tabular}{|c|c|c|c|c|}
\hline & \multicolumn{2}{|c|}{ Grupo salina } & \multicolumn{2}{|c|}{ Grupo diatrizoato } \\
\hline & Pré & Pós & Pré & Pós \\
\hline Machos & $0,45 \pm 0,09$ & $0,58 \pm 0,03$ & $0,56 \pm 0,03$ & $0,87 \pm 0,08^{*}$ \\
\hline Fêmeas & $0,61 \pm 0,1$ & $0,63 \pm 0,09$ & $0,46 \pm 0,16$ & $0,78 \pm 0,13^{\dagger}$ \\
\hline
\end{tabular}

* $p<0,05$ pós versus pré-tratamento (salina: $p=0,107$; diatrizoato: $p=0,001$ ); ${ }^{\dagger} p<0,05$ pós versus prétratamento (salina: $p=0,421$; diatrizoato: $p=0,004$ ).

Tabela 2 Valores de creatinina (média \pm desvio-padrão), pré e pós-tratamento, nos grupos que receberam, além do diatrizoato de sódio, n-acetilcisteína ou alopurinol ou as duas drogas.

\begin{tabular}{|c|c|c|c|c|c|}
\hline \multicolumn{2}{|c|}{ N-acetilcisteína } & \multicolumn{2}{|c|}{ Alopurinol } & \multicolumn{2}{|c|}{ N-acetilcisteína + alopurinol } \\
\hline Pré & Pós & Pré & Pós & Pré & Pós \\
\hline $0,50 \pm 0,07$ & $0,72 \pm 0,11^{*}$ & $0,54 \pm 0,18$ & $0,64 \pm 0,15$ & $0,55 \pm 0,11$ & $0,72 \pm 0,18^{*}$ \\
\hline
\end{tabular}

* $p<0,05$ pós versus pré-tratamento ( $n$-acetilcisteína: $p=0,0002$; alopurinol: $p=0,688$; n-acetilcisteína + alopurinol: $p=0,018$ ).

tilcisteína e alopurinol não impediu o aumento significativo nos níveis séricos de creatinina após o tratamento com diatrizoato $(0,55 \pm 0,11$ e $0,72 \pm 0,18 ; t=2,861$; valor- $p=0,018$ ) (Tabela 2 ).

\section{DISCUSSÃO}

A NIC é freqüente, especialmente em pacientes diabéticos com creatinina aumentada, e apresenta taxa de mortalidade acima de $30 \%{ }^{(4,5)}$. A compreensão dos mecanismos envolvidos na NIC é fundamental para que medidas protetoras possam ser adotadas nesta potencialmente prevenível causa de insuficiência renal aguda ${ }^{(8)}$. A partir das evidências de que espécies reativas do oxigênio estão envolvidas na gênese da NIC, diversos autores têm avaliado, em estudos clínicos e experimentais, o papel protetor de diferentes "varredores" de radicais li$\operatorname{vres}^{(13,14,20)}$.

Em seu pioneiro estudo com a n-acetilcisteína, Tepel et al. demonstraram que a associação da droga à hidratação confere maior proteção renal aos radiocontrastes do que a hidratação isoladamente em pacientes com dano renal prévio ${ }^{(20)}$. Resultados conflitantes foram encontrados por diversos autores ${ }^{(15-19,23-25)}$, o que poderia ser justificado, em parte, por diferenças na população estudada e/ou na diversidade de protocolos de administração da n-acetilcisteína. Além disso, existem evidências indiretas de que a n-acetilcisteína interfere no transporte tubular da creatinina e diminui seus níveis séricos. Hoffmann et al. compararam as concentrações de creatinina e de cistatina $\mathrm{C}$ antes e após 4 horas e 48 horas da administração oral de n-acetilcisteína (600 mg, quatro doses com intervalos de 12 horas) em 50 voluntários com função renal normal que não receberam radiocontraste. Houve diminuição significante da média da creatinina sérica, enquanto a cistatina $\mathrm{C}$, um marcador da função renal que não é afetado pelo transporte tubular, não mostrou variação ${ }^{(26)}$. Ainda que o uso da n-acetilcisteína seja recomendado em pacientes de risco, o valor dessa droga na prevenção da NIC permanece controverso ${ }^{(27,28)}$.

A nefropatia pelo uso de radiocontrastes é difícil de ser induzida em animais normais, porém pode se desenvolver em animais nos quais estejam presentes condições que predisponham à isquemia renal ${ }^{(3)}$. Assim, modelos experimentais com cães, coelhos e, principalmente, ratos submetidos a depleção de sódio, uso de indometacina, uninefrectomia e inibidores da síntese de óxido nítrico, isoladamente ou em associação, têm sido usados ${ }^{(\mathbf{3 , 2 1 , 2 2})}$. No presente estudo, os animais foram submetidos a nefrectomia unilateral e a privação de água, o que os tornou sensíveis à nefrotoxicidade pelo diatrizoato de sódio, um agente de contraste radiológico hiperosmolar.

O alopurinol é um inibidor da xantina oxidase, utilizado clinicamente no tratamento dos estados hiperuricêmicos há mais 
de 50 anos. Em 1988, Katholi et al. estudaram e demonstraram o papel do stress oxidativo na nefropatia induzida por contraste em 39 pacientes com magnésio sérico normal ou baixo, randomizados para receber alopurinol ou placebo antes de serem submetidos a angiografia coronariana ${ }^{(\mathbf{1 3})}$. Mais recentemente, o alopurinol tem sido estudado em condições em que a ocorrência de dano tecidual envolve espécies reativas do oxigênio, especialmente na síndrome de isquemia-reperfusão ${ }^{(\mathbf{2 1 , 2 2})}$ e nefropatia pelos aminoglicosídeos ${ }^{(29)}$, com resultados ainda não-definitivos.

Nosso estudo avaliou a extensão do dano funcional renal por meio da dosagem da creatinina sérica. Mesmo reconhecendo as suas limitações como marcador da função renal, optamos pela creatinina sérica pela sua praticidade e por ser a creatinina o indicador laboratorial utilizado na definição clínica da NIC.

Os animais uninefrectomizados e submetidos a depleção de volume receberam diatrizoato de sódio, por via venosa, e apresentaram elevação significativa da creatinina sérica, sem diferença estatisticamente significante entre machos e fêmeas na intensidade do dano renal. Nós já havíamos demonstrado que a nefrotoxicidade pela gentamicina em ratos é mais intensa no sexo feminino ${ }^{(\mathbf{3 0})}$. Mais recentemente, Habeb et al. apontaram o sexo feminino como possível fator de risco para a NIC ${ }^{(\mathbf{1 0})}$. Embora o stress oxidativo desempenhe papel importante, tanto na necrose tubular aguda pelos aminoglicosídeos quanto na NIC, os mecanismos fisiopatológicos envolvidos são diferentes. Ainda que não apontem para diferenças na gravidade da NIC entre animais do sexo masculino e do sexo feminino, nossos resultados não são suficientes para descartar definitivamente a possibilidade de que as fêmeas sejam mais vulneráveis aos radiocontrastes.

A administração de n-acetilcisteína não foi capaz de prevenir a elevação da creatinina neste estudo, embora tenhamos utilizado doses elevadas da droga. Outros estudos com protocolos semelhantes também falharam em demonstrar este efeito protetor da n-acetilcisteína ${ }^{(19)}$. A fisiopatologia da NIC é complexa, envolvendo distúrbios hemodinâmicos renais e citotoxicidade direta, e a participação das espécies reativas do oxigênio parece ser apenas um dos fatores envolvidos, o que poderia explicar os resultados conflitantes nos estudos clínicos e experimentais com a utilização deste composto.

Os animais que receberam diatrizoato de sódio e alopurinol não apresentaram elevação estatisticamente significante da creatinina, o que sugere um efeito protetor do alopurinol na função renal. Os meios de contraste radiológico aumentam a excreção renal de uratos, por inibirem a sua reabsorção. Tem sido sugerido que obstrução tubular por uratos, oxalatos e proteínas anormais possa ter importante papel na patogênese da NIC ${ }^{(13)}$. Além disso, conforme demonstrado por Bakris et al., a administração de diatrizoato de sódio induz aumento transitório na excreção urinária de proteína de Tamm-Horsfall, mediado em parte por radicais livres do oxigênio danosos para o rim, contribuindo para acentuar o processo obstrutivo intratubular ${ }^{(23)}$. O alopurinol pode ser uma alternativa superior à n-acetilcisteína, por aliar às suas ações antioxidante e hipouricemiante a capacidade de prevenir a obstrução tubular aguda. Um aspecto importante no desenho do presente estudo foi que o alopurinol foi administrado 24 horas antes da infusão do diatrizoato de sódio, para que houvesse tempo para a produção do oxipurinol, principal metabólito ativo do alopurinol e potente inibidor da xantina-oxidase ${ }^{(\mathbf{1 3})}$.

Neste estudo, a associação n-acetilcisteína/alopurinol não foi capaz de evitar o dano renal pelo diatrizoato de sódio. Andrade et al. encontraram maior proteção com a associação Nac/Alo em um modelo de insuficiência renal aguda pós-isquemia/ reperfusão, do que aquele encontrado com as drogas isoladamente ${ }^{(22)}$. Embora nos dois modelos de insuficiência renal aguda haja participação de mecanismos oxidativos, estes parecem ter papel mais importante na injúria renal pós-isquemia/reperfusão, enquanto o dano pela hipóxia tubular na NIC provavelmente é acentuado pela disfunção endotelial e alterações na microcirculação renal.

É sabido que a enzima xantina-oxidase é capaz de gerar radicais superóxidos nos processos de isquemia e existem evidências de que a obstrução tubular após uso de contrastes radiológicos pode acentuar o dano renal induzido por esses compostos Com os relatos recentes apontando a hiperuricemia como um fator de risco para a NIC, os resultados do presente estudo sugerem que o alopurinol pode ser uma alternativa útil na proteção renal nos procedimentos que envolvem o uso de radiocontrastes em pacientes de risco.

\section{CONCLUSÕES}

Neste estudo, o diatrizoato de sódio administrado por via venosa resultou em dano funcional renal, conforme demonstrado pela elevação significativa da creatinina sérica, independentemente do sexo dos animais. $\mathrm{O}$ alopurinol, ao contrário da nacetilcisteína, mostrou-se capaz de proteger a função renal nos animais tratados com diatrizoato de sódio.

\section{Agradecimentos}

Agradecemos a competente ajuda de Valéria Cristina Rocha e de Janaína L. M. Vimeney na fase experimental deste trabalho, especialmente nos procedimentos cirúrgicos.

\section{REFERÊNCIAS}

1. Persson PB. Contrast medium-induced nephropathy [editorial]. Nephrol Dial Transplant. 2005;20 Suppl 1:i1.

2. Voeltz MD, Nelson MA, McDaniel MC, et al. The important properties of contrast media: focus on viscosity. J Invasive Cardiol. 2007;19 Suppl A: 1A-9A.

3. Morcos SK. Contrast media-induced nephrotoxicity - questions and answers. Br J Radiol. 1998; 71:357-65.

4. Levy EM, Viscoli CM, Horwitz RI. The effect of acute renal failure on mortality. A cohort analisys. JAMA. 1996;275:1489-94.

5. Nash K, Hafeez A, Hou S. Hospital-acquired renal insufficiency. Am J Kidney Dis. 2002;39: 930-6.

6. Nikolsky E, Aymong ED, Dangas G, et al. Radiocontrast nephropathy: identifying the high-risk patient and the implications of exacerbating renal function. Rev Cardiovasc Med. 2003;4 Suppl 1:S7-14.

7. Rudnick MR, Goldfarb S, Wexler L, et al. Neph rotoxicity of ionic and nonionic contrast media in 1196 patients: a randomized trial. The Iohexol Cooperative Study. Kidney Int. 1995;47:254-61.

8. Inda Filho AJA. Nefropatia induzida por contraste: podemos prevenir? J Bras Nefrol. 2004; 26:84-95.

9. Baker CSR, Baker LRI. Prevention of contrast nephropathy after cardiac catheterisation. Heart. 2001;85:361-2.

10. Habeb M, Ağaç MT, Aliyev F, et al. Contrast media-induced nephropathy: clinical burden and 
current attempts for prevention. Anadolu Kardiyol Derg. 2005;5:124-9.

11. Toprak O, Cirit M, Esi E, et al. Hyperuricemia as a risk factor for contrast-induced nephropathy in patients with chronic kidney disease. Catheter Cardiovasc Interv. 2006;67:227-35.

12. Grobner T. Gadolinium - a specific trigger for the development of nephrogenic fibrosing dermopathy and nephrogenic systemic fibrosis? Nephrol Dial Transplant. 2006;21:1104-8

13. Katholi RE, Woods WT Jr, Taylor GJ, et al. Oxygen free radicals and contrast nephropathy. Am J Kidney Dis. 1998;32:64-71.

14. Ichikawa I, Kiyama S, Yoshioka T. Renal antioxidant enzymes: their regulation and function. Kidney Int. 1994;45:1-9.

15. Drager LF, Andrade L, Barros de Toledo JF, et al. Renal effects of $\mathrm{N}$-acetylcysteine in patients at risk for contrast nephropathy: decrease in oxidant stress-mediated renal tubular injury. Nephrol Dial Transplant. 2004;19:1803-7.

16. Lopez BL, Snyder JW, Birenbaum DS, et al. Nacetylcysteine enhances endothelium-dependen vasorelaxation in the isolated rat mesenteric artery. Ann Emerg Med. 1998;32:405-10.

17. Briguori C, Manganelli F, Scarpato P, et al. Acetylcysteine and contrast agent-associated nephrotoxicity. J Am Coll Cardiol. 2002;40:298-303.
18. Kay J, Chow WH, Chan TM, et al. Acetylcysteine for prevention of acute deterioration of renal function following elective coronary angiography and intervention: a randomized controlled trial. JAMA. 2003;289:553-8.

19. Durham JD, Caputo C, Dokko J, et al. A randomized controlled trial of $\mathrm{N}$-acetylcysteine to prevent contrast nephropathy in cardiac angiography. Kidney Int. 2002;62:2202-7.

20. Tepel M, van der Giet M, Schwarzfeld C, et al. Prevention of radiographic-contrast-agent-induced reductions in renal function by acetylcysteine. N Engl J Med. 2000;343:180-4.

21. Rhoden EL, Mauri M, Petteffi L, et al. Efeitos do alopurinol sobre a lipoperoxidação de membranas celulares renais na síndrome da isquemia e reperfusão: estudo experimental em ratos. Rev Bras Cir. 1997;87:225-8.

22. Andrade SC, Dezoti C, Shibuya CA, et al. Comparative roles of alopurinol and n-acetylcysteine in the ischemic acute renal failure. J Bras Nefrol. 2004;2:69-75.

23. Bakris GL, Gaber AO, Jones JD. Oxygen free radical involvement in urinary Tamm-Horsfall protein excretion after intrarenal injection of contrast medium. Radiology. 1990;175:57-60.

24. Marenzi G, Assanelli E, Marana I, et al. Nacetylcysteine and contrast-induced nephropathy in primary angioplasty. N Engl J Med. 2006;354: 2773-82.

25. Mouhayar EN, Tadros G, Akinwande AAO. Prevention of contrast-induced renal dysfunction with acetylcysteine in patients undergoing coronary angiography [abstract]. J Am Coll Cardiol. 2002;39(Suppl A):1A.

26. Hoffmann U, Fischereder M, Krüger B, et al. The value of $\mathrm{N}$-acetylcysteine in the prevention of radiocontrast agent-induced nephropathy seems questionable. J Am Soc Nephrol. 2004;15:40710.

27. Birck R, Krzossok S, Markowetz F, et al. Acetylcysteine for prevention of contrast nephropathy: meta-analysis. Lancet. 2003;362:598-603.

28. Alonso A, Lau J, Jaber BL, et al. Prevention of radiocontrast nephropathy with $\mathrm{N}$-acetylcysteine in patients with chronic kidney disease: a metaanalysis of randomized, controlled trials. Am J Kidney Dis. 2004;43:1-9.

29. Smyth BJ, Davis WG. Allopurinol fails to protect against gentamicin-induced renal damage in normotensive and spontaneously hypertensive rats. Nephron. 1994;68:468-72.

30. Carraro-Eduardo JC, Oliveira AV, Carrapatoso $\mathrm{ME}$, et al. Effect of sex hormones on gentamicininduced nephrotoxicity in rats. Braz J Med Biol Res. 1993;26:653-62. 